\title{
A Comparison of Biomimetic Design and TRIZ Applied to the Design of a Proton Exchange Membrane Fuel Cell
}

\author{
Jessica Currie, Keith Fung, A.G. Mazza, J.S. Wallace, L.H. Shu* \\ Dept. of Mechanical and Industrial Engineering, University of Toronto \\ *shu@mie.utoronto.ca
}

\begin{abstract}
The Proton Exchange Membrane (PEM) fuel cell is an attractive energy conversion device that can provide efficient and clean electrical energy. However, limitations in water management can deleteriously affect its conversion efficiency. Overcoming this technological challenge is essential. In this paper, two design methodologies, biomimetic design and TRIZ, were used to find potential solutions to this water management problem. Each design method produced two potential solutions. We found biomimetic design to have more potential for bias by the designer's prior knowledge of biology. This bias can serve to either hinder or help the design process. A notable finding is that biomimetic design and TRIZ can be considered mutually inclusive in the potential solutions generated in this study. This suggests that either design method could have been used to produce the same solutions.
\end{abstract}

\section{Introduction}

A proton exchange membrane (PEM) fuel cell converts the stored chemical energy in a fuel, e.g., hydrogen, into electrical energy. An important and current challenge in PEM fuel cells involves water management, the delicate balance between providing enough water to the membrane for hydration, and removing product water from the cell quickly enough to prevent membrane flooding. Reducing the flooding problem can allow for higher fuel cell operating efficiencies and power densities. To address the water management challenge, biomimetic design and TRIZ (Russian acronym for the theory of inventive problem solving) are applied to identify possible ways to mitigate flooding in the redesign of a PEM fuel cell.

\section{Background}

This section provides background on the two design techniques used in this work, biomimetic design and TRIZ; and briefly describes PEM fuel cells.

\subsection{Biomimetic Design}

In biomimetic design, biological phenomena are studied to generate ideas for solving engineering problems. The classic example of biomimetic design is Velcro, which uses hook structures found on burrs that were observed to attach to fur and clothing. Velcro resulted from the designer's own experience with burrs, inspiring a fastening solution. However, many designers may not know of all relevant biological phenomena that could solve a given problem. Thus, Vakili and Shu (2001) developed a systematic method that searches existing biological knowledge in naturallanguage format to identify relevant biological analogies. The initial corpus, or text, is an undergraduate level biology textbook, Life, the Science of Biology (Purves et al. 2001). Search keywords correspond to verbs that describe the desired function of the solution, and identify biological phenomena that can be used either directly for a design solution or as a starting point for further research into useful biological phenomena.

This method was used to solve problems in design for remanufacture (Vakili \& Shu, 2001; Hacco \& Shu, 2002) and assembly of micro-parts (Shu et al., 2006). To improve the approach, biologically meaningful keywords were generated to bridge different lexicons between engineering and biology (Chiu \& Shu, 2005).

\subsection{TRIZ (Theory of Inventive Problem Solving)} TRIZ, the Russian acronym for "Theory of Inventive Problem Solving" has been shown to significantly increase the number of unique solutions compared to traditional methods such as brainstorming (Okudan et al., 2006). To develop TRIZ, Genrich Altshuller et al. (Altshuller, 1988) studied over 1.5 million patents to identify 39 engineering parameters as commonly improved attributes in innovative designs. Forty principles were generated based on methods used to solve engineering problems in these patents. Many tools are proposed for TRIZ, but the classical contradiction table remains as one of the more powerful tools (Ross, 2006).

To use the contradiction table, a given problem is expressed in terms of the 39 engineering parameters. Pairs of parameters, one of which is degraded in the process of improving the other, are used to identify principles that have been observed to overcome this contradiction. These principles are examined to generate ideas. The contradiction table, parameters, and descriptions of the principles used in this paper are from the text, Product Design Techniques in Reverse 
Engineering and New Product Development (Otto \& Wood, 2001). Although examples for each principle are provided in the text, we did not use these examples as potential analogies, only the description of the principles themselves. We did this to reduce the potential for design by analogy in TRIZ, as we intended to perform design by analogy using biomimetic design, and compare the two approaches.

\subsection{Related Work in Biomimetic Design and TRIZ} Smith and Vincent (2002) recognized a connection between engineering design that seeks to optimize cost versus benefit, and biological systems evolved to optimize metabolic cost versus fitness. Vincent et al. (2002, 2005) are therefore working towards incorporation of biological knowledge into TRIZ. Our work does not seek to combine biomimetic design and TRIZ, but to compare the two design methods.

\subsection{Fuel Cells}

Fuel cells are attractive energy conversion devices. By converting chemical directly into electrical energy, fuel cells achieve higher conversion efficiency $(\sim 50 \%$ and higher) than heat engines that involve intermediate conversions between thermal and mechanical energies. Fuel cells can also produce electricity with a minimal amount of local environmental pollution, e.g., PEM fuel cells that use hydrogen and air will only produce water, electricity, and heat as their products. Of course, the lowest overall pollution possibilities will be realized when the hydrogen gas for fuel can be generated from renewable energy sources, such as solar and wind energy. With these two attractive attributes, fuel cell technology is one of the most promising energy conversion devices for the future. Figure 1 shows a 2-cell PEM fuel cell. Multiple cells are stacked to increase voltage output. Section 2.4.1 defines fuel-cell nomenclature used.

\subsubsection{Fuel-Cell Nomenclature}

Anode: Electrode from which electrons are drawn externally out of the cell.

Bipolar Plates: Cell separation plates that supply reactants, provide electrical and thermal conduction, and provide structural support to the fuel cell. Both sides are channelled.

Cathode: Electrode to which electrons are supplied externally to the cell.

Flooding: Occurs when reaction sites are blocked by water, preventing the reactant from reaching the reaction sites.

GDL: Gas Diffusion Layer - Distributes reactant gas and enhances electrical conductivity between electrode and plate.

MEA: Membrane Electrode Assembly - Includes proton exchange membrane, anode, cathode, and two GDL's.
Nafion ${ }^{\circledR}$ Membrane: A Teflon ${ }^{\circledR}$-like membrane that allows protons to be transported from anode to cathode, while preventing reactants and electrons from crossing.

PEM: Proton Exchange Membrane, most commonly a Nafion ${ }^{\circledR}$ membrane.

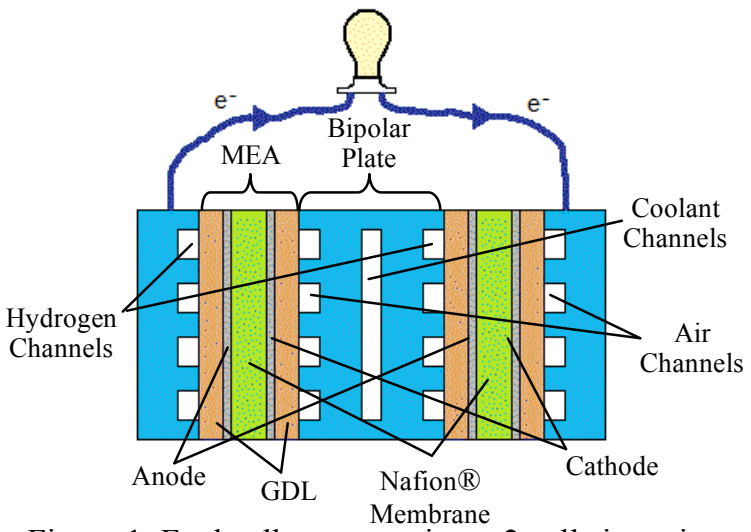

Figure 1. Fuel cell cross section -2 cells in series

\section{Problem Definition: Water Management}

Water management in the design of a PEM fuel cell is very important for two reasons. Firstly, the membrane needs water to conduct protons, a product of the anode reaction, from anode to cathode effectively. For each proton that moves across the membrane, several water molecules are carried across to the cathode side by electro-osmotic drag. This can cause drying of the membrane. However, an excess of liquid water can seriously limit the flow of reactant air/oxygen to the cathode catalyst sites. An excess of water can occur because on the cathode side, water is being formed by reaction as well as carried through the membrane with the proton. Excess water hinders the reactions and decreases the power output. These two major considerations in water management are termed membrane hydration and cathode flooding.

\section{Biomimetic Design}

This section describes two biological analogies identified using the biomimetic search. Descriptions of the biological phenomena, how the phenomena can be related to fuel cells, and potential solutions to the fuel cell water management problem follow.

\subsection{Reproduction in Mushrooms}

The initial keyword, 'distribute', comes from the problem statement of distributing water to achieve membrane hydration at the anode side. Through a series of troponym (more specific form of a verb) relationships in WordNet $\AA$, the keyword 'discharge' was identified and used to search the biology text. The biomimetic search tool returned the following excerpt from Purves et al., 2001: 
"The sexual reproductive structure of basidiomycetes is a basidium: The basidia discharge their spores into the air spaces between adjacent gills, and the spores sift down into air currents for dispersal and germination as new haploid mycelia."

\subsubsection{Biological phenomenon}

Mushrooms are one of the most well-known groups of fungi under the basidiomycete class. Figure 2 shows the basidiomycete structure consisting of a cap sitting on a stalk. The radial gills on the underside of the cap are layers of thin strips of tissue. The spores of basidiomycetes, called basidiospores, equivalent to the seeds of a plant, are the reproductive bodies that can grow into new organisms. On the surface of the gills are tiny protruding stalks called basidia. About two to four basidiospores can be produced on a basidium that, once mature, disperse down into the air current to a different area for germination as new young mushrooms (Purves et al., 2001).

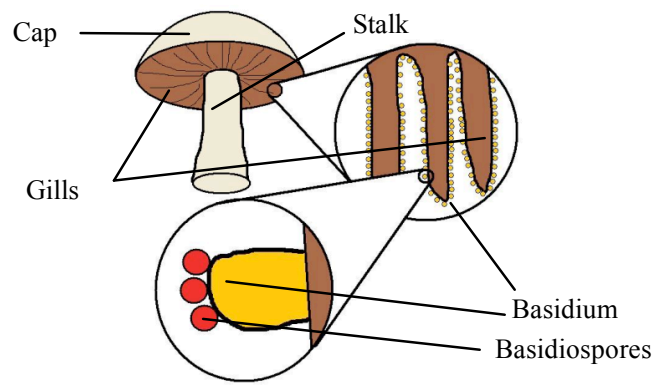

Figure 2. Mushroom Detail

\subsubsection{Domain mapping}

The analogy between the mushroom's reproductive structure and membrane hydration is based on how the basidiomycete discharges its basidiospores through the air spaces between the gills into the air current to carry to suitable germinating sites. Similarly, the anode bipolar plate can be made porous to allow the discharge of water through the pore openings into the moving hydrogen gas. The water is evaporated into the flowing hydrogen gas because of the concentration gradient between the water on the surface of the bipolar plates and the relative humidity of the hydrogen gas. This configuration could provide sufficiently hydrated hydrogen gas throughout the flow field channels to hydrate the membrane.

Bipolar plates, typically made from graphite, which is inherently porous, are rendered impermeable, through plate thickness or sealed with polymer fillers to avoid fuel crossover from anode to cathode (Li, 2006). A typical PEM fuel cell flows coolant within the bipolar plates to remove heat generated from the reactions. If water is used as the coolant and porosity is also retained in the graphite, one can supply water to the anode channels through the pores in the anode plate. The evaporated liquid water will replace water in the hydrogen gas stream lost to the membrane.

\subsubsection{Limitations and advantages}

Minimizing hydrogen leakage into the coolant is desired. Two-phase flow, hydrogen gas and liquid water, will require greater pumping power and also damage the coolant pump. Higher coolant pressure will limit but not eliminate hydrogen diffusion into the coolant due to the concentration gradient. However, the rate of diffusion may be offset by the coolant flow rate from the coolant side to the anode side. Note that the plate on the anode side will be different from the plate on the cathode side, due to the fact that water is removed at the cathode. As a result, the cathode plate must be kept impermeable. The anode-plate thickness may have to be adjusted to achieve the desired water flow rate to the anode from the coolant. In the extreme case where the plate becomes too thin, the channels can then be formed on the GDL instead ( $\mathrm{Li}, 2006)$.

\subsubsection{Existing solution}

After the concept was formulated using biomimetic design, a literature search was performed and a related idea by Peter Schütz (1989) was found. Schütz also uses bipolar-plate porosity to humidify the hydrogen gas, and conducted experiments confirming that the system indeed was able to humidify the hydrogen gas. Schütz's results support that the concept formed using biomimetic design is feasible.

\subsection{Solute Excretion in Plants}

The same initial keyword as in Section 4.1, 'distribute', was expanded using WordNet ${ }^{\circledR}$. However, in this case, the problem considered was distributing water away from the membrane to avoid cathode flooding. Hypernyms (more general forms) of 'distribute', 'transport' and 'conduct', were used in the search for biological phenomena. These searches returned many general biological phenomena that relate to the transport of sap and solutes in plants. Though a particular analogy was not found that inspired a solution in the initial search, it did inspire further research into the more specific mechanisms of plant sap and solute transport. This further research confirmed the relevance of solute excretion in plants.

\subsubsection{Biological phenomenon}

The sap of plants may contain solutes such as salts or heavy metals that enter the plant through the roots from the soil. These can be very harmful to the plant in large quantities and must be removed. The mechanism of solute excretion involves directing solutes by means of relative resistances in plant vessels. In some plant leaves, including maize and 
eucalyptus leaves, the outermost vein consists of one very large vessel. A combination of low resistance due to its large size and rapid evaporation from the surface of the leaf causes solutes to be drawn there. There is no vein sheath (protective layer of cells) on this vessel, so solutes can easily be removed by excretion, dissolved out by rain, and pushed out by positive pressure that exists in the xylem, or plant tissue, at night (Atwell et al., 2001).

\subsubsection{Domain mapping}

The fuel or oxidant gas can be considered analogous to the sap in the plant veins. Likewise, the water in the fuel cell channels can be considered analogous to the solutes in the plant veins. Since solutes can be removed from plant veins by directing them to the area where removal is easily accomplished, by analogy, water could be removed from fuel cell channels by directing it away from where it can do harm, i.e. flood.

In plants, the unprotected large diameter vein provides a large surface area where solutes can be removed from the vein. A similar concept can be used in the fuel cell; however, the phenomenon would occur in the opposite direction because to remove water it must be evaporated into the channels as opposed to out of them. In a fuel cell, a wide channel can provide a large surface area for water to be evaporated into the channel from the membrane.

In addition, the larger channel diameter plant vein provides lower resistance to direct the sap to the location where solutes can be removed. In a fuel cell, lower resistance due to larger width channels could direct oxidant gas to the locations of the fuel cell where water removal is desired.

\subsubsection{Limitations and advantages}

Increasing channel width decreases the inter-channel contact area between the GDL and the bipolar plate, which could hinder current collection. In addition, inter-channel contact area is important for structural support, thus this design solution would need to ensure that there is sufficient support for the membrane.

An important advantage of this solution is that no additional energy input into the fuel cell is required.

\section{TRIZ}

This section will describe two solutions to the fuel cell water removal problem found using TRIZ. We present for each solution: the contradictions, the principle, and how the principle is applied to the fuel cell.

\subsection{Principle 19: Periodic Action}

In a fuel cell, cathode flooding occurs when increased power output produces more water by the cathode-side reaction than can be carried away. When this occurs, the reaction cannot take place and the power decreases rapidly. Therefore there is a conflict between increasing the power and the amount of water that is produced through this increase.

This contradiction can be represented by the TRIZ Generic Engineering Parameters. When we improve power, Parameter 21, the result is an undesired quantity of substance created, Parameter 26 . The TRIZ relationship matrix identifies for this contradiction the following Principles: 4 - Asymmetry, 34 - Discarding and regenerating parts and 19 - Periodic action. A type of asymmetry was already used in the potential solution given in Section 4.2, asymmetry of channel diameter. As for Principle 34, the only part of the fuel cell that this would be applied to, is the water, which we would like to evaporate after it brings hydrogen ions across the membrane, but this is the general goal for any of the potential design solutions. Therefore, Principle 19 - Periodic action, was used.

\subsubsection{Concept generation}

The principle of periodic action states: "Use periodic or pulsed actions, change periodicity" (Otto \& Wood, 2001; Altshuller, 1988). This led to the idea of periodically reversing the flow of air to the cathode side of the fuel cell. Pulsing of the current load was also considered as a possible solution, but a smooth current is desired, so this type of pulsing is not useful.

On the cathode side of the fuel cell, the further along the channel, the more water is contained in the oxidant, decreasing its ability to draw more water out. Periodically reversing the air flow to the cathode side of the fuel cell, especially when high power output is required, could help remedy this situation. When the air near the outlet is unable to carry more water, the flow direction of the air can be reversed, allowing the water to evaporate into the dry incoming air.

\subsubsection{Limitations and advantages}

This solution may cause fluctuation in the power output due to a brief stagnation of the fluid flow during the flow reversal. To ensure that the stack supplies a constant power, a hybrid system, including ultracapacitors or storage batteries, may be required. The ultracapacitors or batteries can be recharged by the fuel cell after the flow reversal.

A possible drawback of this solution is that additional energy is required to reverse the flow of the gas. This loss may be acceptable when weighed against the benefits of reduced flooding.

\subsection{Principle 40: Using Composite Materials}

Flooding on the cathode's reaction sites becomes more severe as current output increases. Since producing more current and protons is desired, this can be 
considered the parameter to be improved, which can be represented by Parameter 26, quantity of substance. The deterioration of the performance due to flooding, on the other hand, can be represented by Parameter 31, harmful side effect. This contradiction leads to the following Principles: 3 - Local quality, 35 - Changing the aggregate state of an object, 39 - Using an inert atmosphere, and 40 - Using composite materials. Since the fuel cell is already heterogeneous, the principle of local quality did not suggest new ideas. As for Principle 35, it can be applied to either water or the electrolyte. Water already changes between liquid to vapour states at various times. A change from solid electrolyte to liquid electrolyte will change the nature of the PEM fuel cell technology. Principle 39 did not lead to new ideas because the atmosphere of the fuel cell is already as inert as possible and measures are already taken to minimize corrosion. Finally, a concept was formed from Principle 40.

\subsubsection{Concept generation}

The resulting concept involves changing the GDL into a composite material. The concept was based on fibre reinforced material, where the fibres' function is to strengthen the material. Similarly, wicks may be applied to GDLs to increase their water removal rate.

GDLs are usually made of carbon paper or cloth. They have randomized pores to allow oxygen to diffuse to the surface of the reaction sites and also provide a means for water to be removed by capillary effects and evaporation. While GDLs may have been coated with a hydrophobic substance to improve water removal, at higher current output, the rate of water removal is insufficient. In order to enhance the cathode GDL's ability to draw water away from the reaction site, wicks can be placed strategically throughout the GDL, as shown in Figure 3.

The material and dimension of the wicks would be chosen to maximize capillary action and the absorption of water. The cathode GDL composite would draw water from the reaction site to the flow field channels in the bipolar plate. Then, water is evaporated to the air as it passes through the channels.

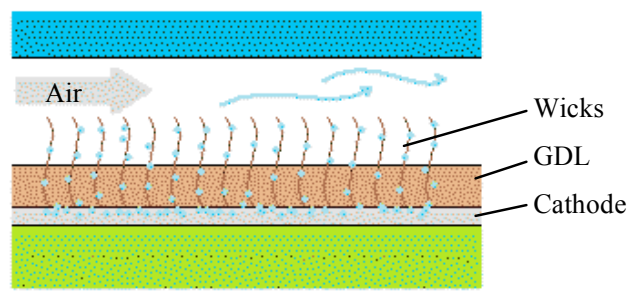

Figure 3. GDL with wicks

\section{Discussion}

This section compares the two design methodologies used to find solutions to the water management problem. The two design methods are compared first in terms of the design processes, and then in terms of the specific solutions that were presented in this paper.

\subsection{Comparison of the Design Methods}

Biomimetic design and TRIZ are compared in the stimuli that inspired the designs, and possible effects of preconceptions.

\subsubsection{Design stimuli}

With biomimetic design, solutions came about as a result of mapping, i.e., identifying possible analogous items in the engineering domain that correspond to those in the biological domain. Thus, we began with the "solution" biological system, of which components were transposed into the engineering system.

With TRIZ, because principles were used, there was less imagery to transfer to the solution. However, this, in fact, was not the case for the composite solution. The visualization of the reinforcing fibres in a composite material was transferred to the solution.

The mapping in biomimetic design tended to be more specific to the part of the fuel cell that was being considered. That is, a given biological phenomenon could not be mapped to different parts of the fuel cell to provide different solutions. TRIZ, on the other hand, has very general principles that can be applied to many different parts of the fuel cell. For example, the periodic action could be applied to the reactant gas flow rate or pressure, the coolant flow rate or pressure or the current draw from the fuel cell.

\subsubsection{Risk of preconceptions and biases}

In biomimetic design, preconceptions and biases can play a major role in the process of selecting biological analogies. For example, with the solute-excretion inspired design, it was obvious that there was a similarity in function between the plant sap distribution system and the fuel cell reactant distribution system. It was this observation that motivated further research into plants. In this case, a useful analogy was found, and so the initial bias was helpful. However it is quite possible that this type of bias can also work against the designer. When one is somewhat familiar with particular biological phenomena, it is easy to have a bias for or against this or other phenomena. Therefore, one should take care to not be overly influenced by such biases. We found this type of bias to be less likely with TRIZ, as we used very abstract design principles to develop concepts. 


\subsection{Comparison of the Generated Concepts}

The plant solute-transport phenomenon provided additional information about solute excretion that was not used in the proposed corresponding solution: at the location of the large vessel there is no protective layer of cells, allowing solutes to be easily removed by excretion, dissolved out by rain, or pushed out by positive pressure at night. Each of these attributes is actually relevant to one of the other solutions presented in this paper. Lacking a protective layer of cells to allow removal is analogous to the "unprotected" porous plate in Section 4.1 that allows water to "excrete" through the pores of the bipolar plate. Also, forced convection along a "positive pressure" gradient is suggested in Section 5.1, where forced flow reversal is used to remove water. These observations were made after all the concepts were developed independently, so the other concepts were not influenced by this particular biological analogy.

TRIZ also identified principles that could have been used to generate some of the other proposed solutions. For example, in Section 5.1 where the principle of periodic action was used to find a solution, Principle 4, asymmetry could also have been used. Asymmetry can also be considered the principle used in Section 4. In that section, it was proposed that the diameter of the fuel cell distribution channels be made larger to facilitate water removal. Also, in that section, different bipolar plates were proposed for anode and cathode, in order to provide hydration to the membrane from the anode side only.

\section{Conclusion}

Water management problems in a fuel cell must be overcome to optimize its performance. Biomimetic design and TRIZ were used to generate concepts to improve water management in a PEM fuel cell. Concepts were generated for hydrating the membrane and for minimizing cathode flooding.

When applying biomimetic design, bias could arise from the designers' prior knowledge of biology. This bias has the potential to either hinder or help the design process. Therefore, extra care should be taken to not to be misled by preconceived biases.

An interesting result of this case study is that biomimetic design and TRIZ were observed to be mutually inclusive in the potential solutions that were found, based on the overlap between the stimuli that were used in the two methods. The TRIZ solutions could have been found using biomimetic design stimuli and the biomimetic solutions could have been found using TRIZ stimuli.

\section{Acknowledgments}

The authors gratefully acknowledge financial support of Natural Sciences and Engineering Research Council through a strategic projects grant, technical support of Hydrogenics, our industrial partner in the project, and Nathan Joos for his helpful discussions.

\section{References}

Altshuller, G.S., 1988, Creativity as an Exact Science, Gordon and Breach Science Publishers Inc., New York.

Atwell, B., P. Kriedmann, C. Turnbull, 2001, Plants in Action: Adaptations in Nature, Performance in Cultivation, MacMillian Publishers Australia Pty. Ltd., South Yarna.

Chiu, I., L.H. Shu, 2005, "Bridging cross-domain terminology for biomimetic design," Proceedings of ASME DETC/CIE, Long Beach, CA. DETC2005-84908 (DTM).

Hacco, E., L.H. Shu, 2002, "Biomimetic Concept Generation Applied to Design for Remanufacture", Proceedings of ASME DETC/CIE, Montreal, QC, Canada, DETC2002/DFM-34177.

Li, X., 2006, Principles of Fuel Cell, Taylor \& Francis Group, New York.

Okudan, G.E., M. Ogot, R. Shirwaiker, 2006, "An investigation on the effectiveness of design ideation using TRIZ," Proceedings of ASME DETC/CIE, Philadelphia, PA, DETC2006-99483.

Otto, K.N., K.L. Wood, 2001, Product Design Techniques in Reverse Engineering and New Product Development, Prentice-Hall, Inc. New Jersey, USA.

Purves, W.K., D. Sadava, G.H. Orians, H.C. Heller, 2001, Life, The Science of Biology, 6/e, Sinauer Associates, Sunderland, MA.

Ross, V.E., 2006, "A Comparison of Tools Based on the 40 Inventive Principles of TRIZ," The TRIZ Journal, 2006. [Online] Accessed: January 15, 2008. Available: http://www.triz-journal.com/archives/2006/11/01.pdf.

Schütz, P., 1989, "Prevention of Membrane Dehydration in PEM Fuel Cells," Proceedings of the Symposium on Fuel Cells, Eds. White, R.E., Appleby, A.J., The Electrochemical Society, Inc., PVP-Vol. 89(14): 87-93.

Shu, L.H., H. Hansen, A. Gegeckaite, J. Moon, C. Chan, 2006, "Case Study in Biomimetic Design: Handling and Assembly of Microparts," Proceedings of ASME DETC/CIE, Philadelphia, PA, DETC2006-99398 (DTM).

Smith, C.W., J.F.V. Vincent, 2002, "Biomimetics: Technology Transfer from Biology to Engineering," Philosophical Trans. of the Royal Society: Mathematical, Physical and Engineering Sciences, 360:15-157.

Vakili, V., L.H. Shu, 2001, "Towards Biomimetic Concept Generation," Proceedings of ASME DETC/CIE, Pittsburgh, PA, DETC2001/DTM-21715.

Vincent, J., D. Mann, 2002, "Systematic Technology Transfer from Biology to Engineering," Philosophical Trans. of Royal Society: Physical Sciences, 360: 159-173.

Vincent, J., 2005, "Deconstructing the design of a biological material," Journal of Theoretical Biology, 236: 73-78. 